\title{
Survey of Related Technologies for Improved Spectrum Efficiency
}

\author{
${ }^{1}$ B.S. Olanrewaju and ${ }^{2} \mathrm{O}$. Osunade \\ ${ }^{1,2}$ Dept of Computer Science, University of Ibadan, Nigeria.
}

\begin{abstract}
The most noted effect of rapid growth in wireless networks in recent years has been the issues of spectrum usage and management because of its importance in wireless communication as the medium of data transmission. Efficient utilization of spectrum has been the major concerns in spectrum management over recent years because of the notion that radio spectrum is scarce, finite, and non-replicable. New technologies find it difficult to be allocated frequencies for their operations because most usable frequencies have already been allocated to licenced users while the unlicenced spectrum bands are becoming overcrowded. Researches have however shown that some of the allocated spectrum bands are not been used at some particular time in a particular location. The development of technologies like $4^{\text {th }}$ generation $(4 G)$ technology, Ultra Wide Band (UWB) techniques, Software-defined Radios (SDRs), and Cognitive Radios (CRs) help to improve the overall utilization of the limited spectrum. This paper surveyed these technologies and their impact in improving spectrum efficiency in terms of spectrum management and utilization. The paper concluded that dynamic spectrum access (DSA) technologies have greater impact when compare with other technologies. Cognitive Radios in particular have the potentials to substantially improve spectrum efficiency by opportunistically using spectrum. Spectrum efficiency in this context means that a large number of users are able to use available spectrum.
\end{abstract}

Keywords: Dynamic Spectrum Management, $4 G$ Technology, Software Radio, Cognitive Radio

\section{Introduction}

Electromagnetic spectrum is the entire range of electromagnetic radiation that propagates as waves in space and it is the basis for all telecommunications channels, both wired and wireless (Williams \& Sawyer, 2003). Electromagnetic spectrum consists of electric waves, radio frequency, infrared, visible light, ultra-violet, $\mathrm{X}$-rays, gamma rays, and cosmic rays. Radio frequency spectrum, in the middle of electromagnetic spectrum is of importance in data communications and this is what is generally referred to as spectrum in telecommunications. It covers a frequency range of about $10 \mathrm{KHz}$ to $300 \mathrm{GHz}$ but most wireless applications operates between $30 \mathrm{MHz}$ and $30 \mathrm{GHz}$ (Cave et al, 2007; Goldsmith, 2005). The usage of radio spectrum and the regulation of radio emissions are coordinated by both national and international regulatory bodies but each country is at liberty to manage spectrum as it wishes so far it portends no interference risks to other countries (Cave et al, 2007).

A large and growing part of the world's output relies upon the use of spectrum for large varieties of things. According to (Steenkiste et al, 2009), wireless technology is proliferating rapidly with over 3 billion wireless devices (most of which are cell phones and mobile computers) in use today, and the number is expected to increase to approximately 100 billion by the year 2025 . With this proliferation and the fact that Dirk-Oliver (2009), describes radio spectrum as a scarce, finite natural resource which can neither be created nor destroyed and also as a competitive public good. The key purpose of spectrum management is to maximize the value that society gains from the radio spectrum by allowing as many efficient users as possible while ensuring that the interference between different users remains manageable (Cave et al, 2007).

Spectrum management as stated by Taylor (2003), entails delivering many information communications technology (ICT) services that requires the scarce and finite resource on a competitive basis, complex planning to ensure efficient use of it, and frequency allocation, assignment, monitoring and ensuring interference free operations. Spectrum manager provides each user with the right to transmit on a particular frequency over a particular area, typically in the form of a licence. In a licenced spectrum, only the licensee has the right to use the spectrum in that particular area. Spectrum allocation is necessary in order to ensure interference free operation for each radio service. A few bands designated as unlicenced spectrum, allow users to share spectrum but with restriction to limit the consequences of interference (Cave et al, 2007; Peha, 2008). Spectrum allocation is necessary in order to ensure interference free operation for each radio service however, most allocated spectrum sits idle at any given time (Peha, 2008; Danda \& Gonjun, 2011; Mark, 2007). When 
exclusive right (licenced spectrum) is given to a particular user to operate in a particular frequency, some sort of inefficient usage of the spectrum is inevitable. This is because the spectrum lies idle when the licensee is not using the spectrum and this limit the introduction of new technologies by supposedly shortage of spectrum which in much of the time sits idle (Cave et al, 2007). Unlicenced spectrum opens up the possibilities of better usage of spectrum by allowing sharing among different users (Steenkiste et al, 2009). But this sharing of the unlicenced spectrum also known as open spectrum sharing must be properly coordinated to ensure that there is no reduction in the quality of service due to interference of multiple users.

\section{Existing Spectrum Management Policies}

As demand grows for spectrum utilization, there is need for spectrum management to avoid the possible interference between different users that might render their systems unusable when transmitting at the same time, on the same frequency and sufficiently close distance (Cave et al, 2007). In managing spectrum, users are allowed to use spectrum using a licensing policy in either different bands or same band in a significantly different location in a way that will not lead to unbearable interference through allocation and assignment. Spectrum allocation means deciding the use of a band for a particular service while deciding the users or organization to use the band for its services is spectrum assignment.

Spectrum regulators or managers are at two major levels namely, international and national. At the international level, the usage of radio spectrum is managed by the International Telecommunications Union, Radiocommunication sector (ITU-R), to ensure international usage of spectrum in a way that it will not infringe on the right or interest of member countries (Taylor, 2003). At the national level, different countries established commissions and ministries to oversee the affairs of spectrum. National regulators manage the resource only for that country in a way that there will not be conflict of interest among users within the territory of the nation. According to Deepak (2002), ITU at the World Radiocommunication Conferences allocates spectrum frequencies on a regional basis and for different types of services for the use of various countries. The ITU set out high level guidance which national bodies must adhere to in setting more detailed policy (Cave et al, 2007). The spectrum managers is to decide the use of a particular band and which users are allowed to transmit in the band thereby keeping a tight regulatory control over the use of the spectrum and ensuring that excessive interference does not occur between neighbouring services (Cave et al, 2007).

ITU stated three key elements of spectrum management which are spectrum standards, spectrum licensing and spectrum planning.

i. Spectrum Standards: This defines the most efficient way of managing interference.

ii. Spectrum Licensing: This defines rights and obligations of spectrum users. A spectrum band could either be licenced or unlicenced. A licenced spectrum is when spectrum managers provide each user with the exclusive right to transmit on a particular frequency over a particular area. Any band of spectrum that spectrum managers allowed users to transmit with no one given exclusive right is unlicenced spectrum. The use of unlicenced spectrum in an uncoordinated manner is ensured by transmitting at a relatively low power to cover a small distance and thereby reduce the probability of any interference among users (Cave et al, 2007). Examples of unlicence services include cordless phones and wireless LANs such as WiFi. One of the advantages of unlicenced spectrum is that the deployment of services can be fast and inexpensive because there is no licensing procedure; however lack of motivation to conserve the share spectrum is a disadvantage (Peha, 2000).

iii. Spectrum Planning: This defines a framework for minimizing interference. It involves the allocation of spectrum both at international and national levels. The methods for frequency planning are typically based on knowledge about frequency characteristics, output power, and sensitivity levels for the systems of interest (Berggren et al., 2004).

Cave et al (2007) listed the shortcomings of the current system to include idle spectrum when allocated band is not used by intended services, differing valuation of spectrum usage by services e.g. cellular and fixed wireless, difficulty of new applications or technologies in gaining access to spectrum e.g. Mobile TV systems, and non-optimization of spectrum usage by applications such as aviation radar which have been granted free spectrum. The existing policies of spectrum management which are based on static spectrum allocation for a specific technology and service controlled by regulation agencies, has become unreasonable to use for some technologies like wireless personal communication regarding economical and technical considerations (Hamid, 2008).

\section{Dynamic Spectrum Management}

Dynamic spectrum management involves the planning, assignment and allocation of spectrum for efficient use. Dynamic spectrum management (DSM) is therefore the application of advanced technology to the design of radios to autonomously and dynamically optimize their communication settings with respect to the spectrum utilization status which include both the channel and the transmissions of neighboring systems. The 
most important aspect of DSM is DSA. Dynamic spectrum access sharing could be between primary users (PUs) and secondary users (SUs) (i.e. licence and unlicence users respectively) or among different secondary users in open spectrum band. In order to improve the efficiency of spectrum, radio systems are allowed to coexist in the same spectrum through dynamic spectrum access. Effective spectrum utilization is achieved when one device transmits while others are idle.

The basic premise of DSA is that spectrum licence holders, known as primary users, should allow other devices, known as, secondary users to transmit in their frequency bands when they are not using them, on a non interference basis, for example, idle TV spectrum can be reused for regional broadband access, which is the goal of IEEE 802.22 (Clancy, 2007). There are two basic dynamic spectrum access approaches, overlay and underlay approach. In the overlay approach, the SUs access the portion of the spectrum that is not used by PUs as a result; there is virtually no interference to the PUs. In the underlay approach, the SUs access the network by spreading their signals over a wide frequency band. The underlay approach imposes severe constraints on the transmission power of SUs. Operating below the noise floor of PUs, the SUs are allowed to interfere with PUs up to a certain tolerable level.

The challenges of DSA include developing wireless devices that can operate in different frequency bands, protecting the right of licence holders and maintaining service quality (Steenkiste et al., 2009). To achieve the goals of DSA, systems involves in spectrum sharing must be able to know whether to transmit or not in order not to cause interference with systems coexisting in the network. With emerging technologies, opportunistic access is becoming more practical, but significant research challenges remain (Peha, 2007). The 5 $\mathrm{GHz}$ unlicenced frequency band is a candidate for a large set of radio services, and is one of the unlicenced frequency bands that may be efficiently used only with established spectrum etiquette (Xing, 2006). However, as stated by Akyildiz et al. (2008), the capability to share the wireless channel with licenced users in an opportunistic manner can be realized through spectrum management functions that address four main challenges: spectrum sensing, spectrum decision, spectrum sharing, and spectrum mobility.

Spectrum Sensing: This is the ability of secondary users to monitor available spectrum bands to detect idle spectrum known as white space or spectrum hole. According to Steenkiste et al. (2009), spectrum sensing is a challenging problem and it remains an important area of investigation by the wireless research community.

Spectrum decision: Based on the spectrum availability, secondary users can allocate a channel but require the capability to decide which spectrum band among the available bands is the best (Akyildiz et al., 2008).

Spectrum Sharing: This is the coexistence of different radio systems in the same spectrum where one device may transmit, while others in the area are idle (Xing et al., 2006). In open spectrum sharing (OSS), where every system has the same priority for accessing the spectrum resource, heterogeneous systems with different channel bandwidth sizes, coexist in a common spectrum (Wang et al., 2010).

Spectrum Mobility: This is the ability of a secondary user to vacate a spectrum when the presence of a primary user is noticed and thereby find alternative vacant portion of the spectrum for continuous transmission (Akyildiz et al., 2008). That is, switching to another available channel when a licenced primary user appears (Wang \& Wang, 2010).

\section{New Technologies to Improve Spectral Efficiency}

Prevention of interference between systems by giving each system exclusive access to a block of spectrum leads to spectrum idleness whenever such a system is not transmitting because no other systems can make use of it (Peha, 2008). Adopting new technologies that can use spectrum more efficiently where economically viable is important to solving the supposed spectrum scarcity in wireless communication. New technologies to improve spectral efficiency include the introduction of higher modulation rates, antenna technology, ultra wideband techniques, $4 \mathrm{G}$ technologies and dynamic spectrum access technologies.

\section{Modulation Technology}

Orthogonal frequency-division multiplexing (OFDM): is a method of encoding data on multiple carrier frequencies using frequency-division multiplexing (FDM) scheme as a modulation method (Zemen, 2008; Soltani, 2009). OFDM as the modulation method of choice for $4 \mathrm{G}$ technologies is spectrally efficient and it mitigates the severe problem of multi-path propagation which means that more data can be transmitted faster in a given bandwidth (Wimax Forum, 2006). OFDM is not only a great modulation method, it also can provide multiple-access to a common bandwidth or channel to multiple users. OFDM seems to be the best modulation technique for now and it is even better with multiple-input multiple-output (MIMO) antenna technology (Frenzel, 2009). Orthogonal frequency-division multiple-access (OFDMA) and single-carrier frequencydivision multiple-access (SC-FDMA) are the multiple-access versions of OFDM where OFDMA is for downlink and SC-FDMA is for uplink (Soltani, 2009). 


\title{
4G Technology
}

$4 G$ Technology is basically the extension of the $3 \mathrm{G}$ technology functionalities to produce more bandwidth and services. 4G technology should support at least 100 Mbps peak rates in full-mobility wide area coverage and $1 \mathrm{Gbps}$ in low-mobility local area coverage. The two main 4G standards are 3GPP-LTE and IEEE $802.16 \mathrm{~m}$ (WiMAX)

3GPP Long Term Evolution (LTE): The 3rd Generation Partnership Project (3GPP) Long Term Evolution (LTE) specification is a technology that offers enhancements to $3 \mathrm{G}$ to meet the needs of $4 \mathrm{G}$ systems as it will be able to use the same infrastructure for its functions and aimed at providing better quality of service. LTE, which is also known as Evolved UMTS Terrestrial Radio Access (e-UTRA), provides new physical layer concepts and protocol architecture for Universal Mobile Telecommunications System (UMTS) (Rohde \& Schwarz, 2008). UMTS is a $3 \mathrm{G}$ technology that supports maximum theoretical data transfer rates of $45 \mathrm{Mbps}$ but which is upgraded by the 3GPP Long Term Evolution project to 4G speeds of 100 Mbps downlink and 50 Mbps uplink, using a next generation air interface technology E-UTRAN, based upon OFDM (Motorola 2007). LTE Evolved UMTS Terrestrial Radio Access Network (E-UTRAN) offers high data speed and improved bandwidth for network service providers as its specification provides down-link peak rates of $300 \mathrm{Mbit} / \mathrm{s}$ and uplink peak rates of $75 \mathrm{Mbit} / \mathrm{s}$.

4G access technologies are expected to improve spectrum efficiency considerably by providing much more capacity in smaller spectrum allocations (Haslam, 2011). The LTE standard can be used with many different carrier bandwidths of $1.4,3,5,10,15$, and $20 \mathrm{MHz}$ resulting to a sort of spectrum flexibility. The spectral efficiency in the LTE will be 2 to 4 times of that of 3G (Motorola 2007).

Worldwide Interoperability for Microwave Access (WIMAX-802.16m): Similar to WI-FI but offers higher speeds over greater distances and for a greater number of users. A WiMAX system consists of a WiMAX tower and a WiMAX receiver. WiMax operates in a non-line-of-sight service in the frequency range of $2 \mathrm{GHz}$ to 11 $\mathrm{GHz}$ or line-of-sight service using higher frequencies in the range reaching $66 \mathrm{GHz}$ with better QoS and more bandwidth. WiMAX share similar performance capabilities with LTE as both technologies exploit similar wireless and IP design techniques to approach the maximum spectral efficiencies defined by Shannon's Law (Motorola, 2007).

WiMAX, is based on an RF technology OFDM, which is a very effective means of transferring data when carriers of width of $5 \mathrm{MHz}$ or greater can be used but below $5 \mathrm{MHz}$ carrier width, current CDMA based $3 \mathrm{G}$ systems are comparable to OFDM in terms of performance(Omerovic, 200x ). License bands in $2.3 \mathrm{GHz}, 2.5$ $\mathrm{GHz}$ and $3.5 \mathrm{GHz}$ and $5.8 \mathrm{GHz}$ and unlicence bands in $2.4 \mathrm{GHz}$ and $5 \mathrm{GHz}$ have been chosen for the operation of WiMax (Wimax Forum, 2006). To take best advantage of the benefits provided by WiMAX systems, large block spectrum assignments are most desirable. This enables systems to be deployed with large channel bandwidths, flexible frequency re-use and with minimal spectral inefficiencies for guard-bands to facilitate coexistence with adjacent operators. (Omerovic, 200x ).

\begin{abstract}
Antenna Technology
Multiple-input and multiple-output (MIMO): This is an antenna technology for wireless communications in which multiple antennas are used at both the transmitter and the receiver to improve communication performance. With the use of multiple antenna technologies the goals of $4 \mathrm{G}$ systems such as high rate, high reliability, and long range communications are being achieved. A distinct problem in conventional wireless communications where a single antenna is used at the source and another single antenna is used at the destination is multi-path effect which causes problems such as fading and intermittent reception. The use of two or more antennas, along with the transmission of multiple signals (one for each antenna) at the source and the destination, eliminates the trouble caused by multi-path wave propagation, and can even take advantage of this effect (Bliss et al, 2005). MIMO combined with orthogonal frequency-division multiplexing (OFDM) or with Orthogonal Frequency Division Multiple Access (OFDMA) modulation, effectively handles the problems created by multi-path channel. MIMO technology has aroused interest because of its possible applications in digital television (DTV), wireless local area networks (WLANs), metropolitan area networks (MANs), and mobile communications.
\end{abstract}

Smart antenna: This is one of the most promising technologies that will enable a higher capacity in wireless networks by effectively reducing multi-path and co-channel interference. Smart antennas employ a set of radiating elements arranged in the form of an array (Winters, 2003).

A type of smart antenna with spatially separated directional beams lead to increase in the possible reuse of a frequency channel by reducing potential interference and also increases the range. Another type, switched beam antenna has a switching mechanism that enables it to select and then switch the right beam which gives the best reception for a mobile user. 
Smart antennas radiate energy only in the desired direction while ordinary antennas radiate energy in all directions leading to a waste of power. Therefore less power is required for radiation at the base station with smart antenna. Reduction in transmitted power also implies reduction in interference towards other users.

\section{Ultra Wideband Techniques}

Ultra wideband is a wireless technology for transmitting large amounts of digital data over a wide spectrum of frequency bands greater than $500 \mathrm{MHz}$ with very low power of less than 0.5 milliwatts up to a distance of 10 meters or 30 feet (Intel, 2005). Ultra wideband can be compared with another short-distance wireless technology, Bluetooth, which is a standard for connecting handheld wireless devices with other similar devices and with desktop computers.

UWB technology operates by sending billions of pulses across a very wide spectrum of frequency in the region of several $\mathrm{GHz}$ through a transmitter while the corresponding receiver translates the pulses into data (Intel, 2005). UWB's combination of larger spectrum, lower power and pulsed data improves speed and reduces interference with other wireless spectrum (Intel, 2005). UWB complements existing longer range radio technologies such as Wi-Fi, WiMAX, and cellular wide area communications that bring in data and communications from the outside world.

In the United States, the Federal Communications Commission (FCC) has mandated that UWB radio transmissions can legally operate in the range from $3.1 \mathrm{GHz}$ up to $10.6 \mathrm{GHz}$ (Fig.1), at a limited transmit power of $-41 \mathrm{dBm} / \mathrm{MHz}$ (Fig.2).

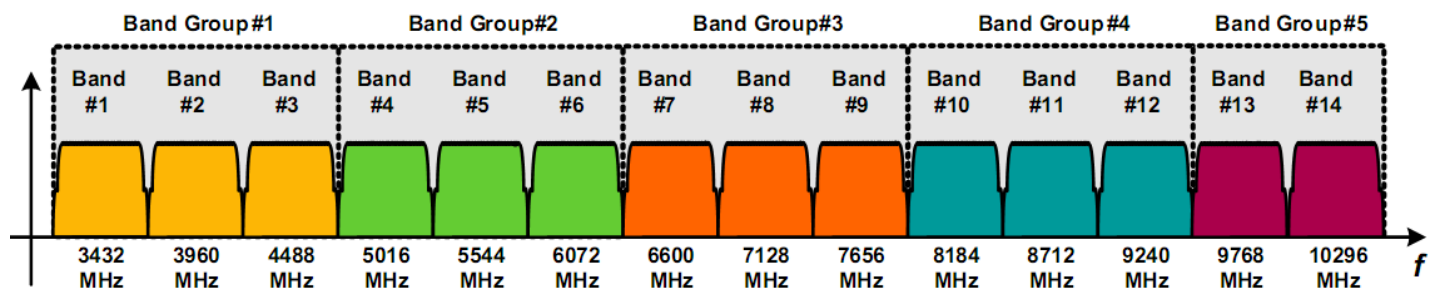

Figure 1: Multi-Band OFDM Source: ECMA International, Ecma/GA/2005/038

UWB uses multi-band OFDM where spectrum is divided into groups of band as shown in figure 1 to separate applications. Longer range applications use band groups \#1 and \#2, while shorter range applications use band groups \#3 and \#4 (Ecma International, 2005).

UWB applications co-exist with the unlicensed National Information Infrastructure (U-NII) bands as shown in figure 2 in an underlay approach with the transmit power set at a reasonable low level of -41 $\mathrm{dBm} / \mathrm{Mhz}$ to avoid interference.

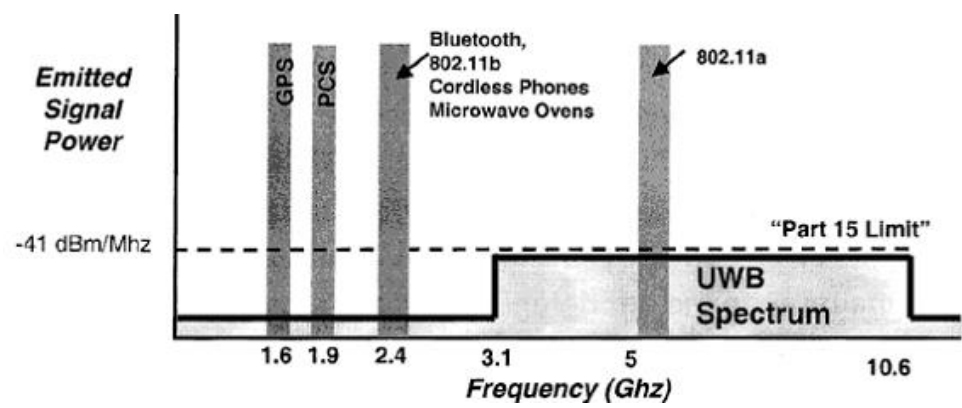

Figure 2: UWB Spectrum Co-existing in Underlay Approach Source: Ahmad et al, 2003.

\section{Dynamic Spectrum Access Technologies}

Radios based on DSA include software defined radios (SDRs) or simply software radios, Cognitive radios (CRs) and a variant of CR is DARPA's XG developed by the Defense Advance Research Projects Agency (DARPA). These radios employ several advances in technology to use spectrum more intensively and efficiently by spectrum sensing and sharing which is very important in DSA.

\section{$\underline{\text { Software Define Radio }}$}

SDR which was conceived by Joe Mitola in 1991 following the advances in processors, RF technology, and software, is a radio system in which the radio functions like modulation/demodulation, encoding/decoding, etc. are handled in software. The SDR Forum defined SDR as radio in which some or the entire physical layer 
functions are software defined. The transmitter generates the radio functions while the receiver recovers the signal. Both the transmitter and the receiver actions are performed in software. The advances in SDR has enabled the development of flexible and powerful radio interfaces for supporting spectral agility that is, dynamic selection of spectrum (Xing et al., 2006). A single SDR can be used in any mode, anywhere in the world by simply selecting and launching the requisite computer program. For example, a single SDR transceiver capable of playing the roles of cordless telephone, cell phone, wireless fax, wireless e-mail system, pager, wireless videoconferencing unit, wireless Web browser, Global Positioning System (GPS) unit, and other functions, is operable from any location on the surface of the earth (SearchNetworking, 2000).

Since 2006 when the evaluation of the adoption of SDRs technologies was conducted by SDR forum, the technologies have been successfully deployed in defense applications as SDR currently finds application in the US military's Joint Tactical Radio System (JTRS) to produce radios that provide flexible and interoperable communications through the use of SDR systems. One of the first public software radio initiatives was a U.S. military project named SpeakEasy with the primary goal of using programmable processing to produce a radio for the U.S. Army which could operate from $2 \mathrm{MHz}$ to $2 \mathrm{GHz}$ (Lackey, 1995).

\section{DARPA's Next Generation (XG)}

The neXt Generation program or XG is a spectrum access technology developed by the Defense Advance Research Projects Agency (DARPA), to produce a set of advanced technologies for dynamic spectrum access, including spectrum measurement techniques, policy based controls and XG behaviors resulting into implementation (Kalliola, 2004). DARPA XG Program is not a new radio but a set of advanced technologies for dynamic spectrum access which has software controlled radios, policies and XG policy controls as its components (Marshall, 2005). Important policies that are needed for managing the key aspects of radio behaviors in the program include regulatory policies to avoid interference, military policies to define military needs and quality of service, and radio policies for defining routing techniques, transmission power, etc (Marshall, 2005). XG policy control has policy checking and inference mechanism to ensure conformity to the policy during implementation.

\section{Cognitive Radios}

The term cognitive radio defines the behaviour of radios and the related wireless devices to be sufficiently, computationally intelligent about the radio resources in order to detect and to provide available radio resources and wireless services most appropriate to those needs (Mark Norton, 2007). A cognitive radio is a software radio equipped with sensors and software that allow it to perceive the operating environment and learn from experience. According to Kalliola (2004) the most disruptive spectrum sharing scheme is the Cognitive Radio.

Cognitive radios by design are capable of sensing their environment and thereby make necessary changes in their transmission parameters to use the spectrum when primary users are inactive and to leave when licence users reappears (Steenkiste et al., 2009; Yuguchi, 2008; Narayan, 2008; Clancy, 2006; James, 2006). Cognitive radio is a technology for spectrum sharing that recognizes vacant bands of spectrum and adjusts autonomously its transmission to one of the bands by using SDR technology (Yuguchi, 2008).

Radio functionalities are major challenges in cognitive radio as it requires a considerable amount of intelligence from the transmitting device. Cognitive radio need to detect the presence of primary users in a licenced spectrum and quit the frequency band as quickly as possible if the corresponding primary radio emerges in order to avoid interference to primary users (Kalliola, 2004). From the definition of cognitive radio, Akyildiz et al (2008) highlighted two main characteristics of cognitive radio to be cognitive capabilities and reconfigurability. This means that a cognitive radio will be able to detect multiple idle frequency bands and decides which frequency band to use since it is in most cases unrealistic that the user will be able to pick the right band (Steenkiste et al., 2009). In other word, cognitive radio systems require the four major management functions of spectrum sensing, spectrum decision, spectrum sharing and spectrum handoff or mobility (Wang \& Wang, 2010).

\section{Comparison of the Technologies on Spectrum Efficiency}

In the comparison of impact on spectrum management for efficient utilization, the technologies are rated based on parameters such as data rates delivered, number of simultaneous users, interference mitigation scheme, spectrum agility, and spectrum usage policy.

These parameters are defined as:

Data Rate: This refers to the effect of techniques employed to produce an appreciable

increase in data transmission in bits per second.

Simultaneous Users/Applications: This defines the ability of the technologies to allow higher number of users/applications in a spectrum band to coexist in a way that it will not 
have adverse effect on the transmission qualities of users.

Interference Mitigation: This is the strength of the technologies to control interference

from other external sources during transmission.

Spectrum Agility: This defines the ability of technologies to allow a user to use spectrum

in a licensed spectrum band when the licensee is absent by sensing the idleness of the spectrum and then using it opportunistically.

Spectrum Usage Policy: In sharing spectrum or coexisting with different users, whatsoever technology adopted must not infringe on other's right in the usage of either licensed or open spectrum.

These parameters are rated high, medium, low, and none accordingly for each of the technologies. It is high if a parameter, through the use of a technology has an appreciable impact on spectrum efficiency, medium for average impact, low for negligible impact and none for non implementation of a parameter in a technology.

Table 1 shows the comparison.

Though LTE and WIMAX as 4G technologies have high data rates which permit greater number of simultaneous users, there is no mechanism for dynamic spectrum access. Interference mitigation system is by power control and adaptive fractional frequency reuse. The spectrum agility of $4 \mathrm{G}$ technologies is considered low because the frequency reuses in $4 \mathrm{G}$ have lesser effect when compared with the opportunistic sharing of spectrum in DSA.

Table 1: Comparison of Technologies

\begin{tabular}{|l|l|l|l|l|l|}
\hline Technology & Data Rate & $\begin{array}{l}\text { Number of } \\
\text { Users/ } \\
\text { Application }\end{array}$ & $\begin{array}{l}\text { Interference } \\
\text { Control }\end{array}$ & $\begin{array}{l}\text { Spectrum } \\
\text { Agility }\end{array}$ & $\begin{array}{l}\text { Spectrum } \\
\text { Policy } \\
\text { Measures }\end{array}$ \\
\hline LTE & High & High & Medium & Low & None \\
\hline WIMAX & High & High & Medium & Low & None \\
\hline OFDM/OFDMA & Medium & Medium & Medium & Low & None \\
\hline MIMO & Medium & Medium & Medium & Low & None \\
\hline Smart Antenna & Medium & Medium & Medium & Low & None \\
\hline UWB & High & High & Medium & Low & None \\
\hline SDR & Medium & Medium & Medium & Medium & None \\
\hline XG & Medium & Medium & High & High & High \\
\hline Cognitive Radio & Medium & High & High & High & High \\
\hline
\end{tabular}

OFDMA, MIMO, and smart antenna are enabling technologies needed to achieve and meet the growing needs of more bandwidth for different services and applications in $4 \mathrm{G}$ technologies. They contribute to the high data rate and therefore more users in wireless communications with ability to control multi-path effect and cochannel interference. As there is no implementation of DSA, there is no spectrum policy needed to protect the interest of multiple users.

UWB has a high scalable data rates from $55 \mathrm{Mb} / \mathrm{s}$ to $480 \mathrm{Mb} / \mathrm{s}$ and transmit at low power to ensure reasonable interference control and also allow co-existence with other users. UWB operates in a fixed range from $3.1 \mathrm{GHz}$ up to $10.6 \mathrm{GHz}$ therefore requires minimal spectrum agility with no spectrum policy apart from the FCC power spectral density emission limit, the Part 15 limit that applies to unintentional emitters in the UWB band.

SDR forum does not specify the data rate but its implementation through modifiable software or firmware operating on programmable processing technologies brings the flexibility, cost efficiency and power to drive communications forward. SDR can control interference through antenna technology locking adaptively to a desired signal and rejecting signals from other directions.

Just as SDR, DARPA XG and Cognitive radio research group do not state the data rate to be achieved. But with the vast areas of incorporated technologies in cognitive radio networks which include smart antenna technology, spectrum sensing and measurement, radio signal processing, hardware architectures including software-defined radio (SDR), medium access control (MAC), network discovery and self-organization, routing, adaptive control of mechanisms, policy definition and monitoring, and learning mechanisms, cognitive radio system is poised to improve data rate and spectrum efficiency in particular.

DARPA XG and Cognitive radio have related concepts that build on the technology of software defined radio and this make them better options for spectrum efficient technologies. Although, SDR can be reconfigured in software to allow its operation when moved to a different operating environment, it is not as flexible or intelligent as cognitive radio and DARPA XG which by their own sense their environment and make use of any available idle spectrum. DARPA XG is in fact a variant of cognitive radio but the technology is developed for military application which limits its wide applications. 


\section{Conclusion and Future Work}

Higher modulation techniques, MIMO, Smart antenna are complementary technologies employed by $4 \mathrm{G}$ technologies to achieve significant spectrum efficiency. Even with the contribution of $4 \mathrm{G}$ technologies to spectrum improvement the demand for spectrum usage will still not be met (Haslam, 2011). UMTS forum also predicts a high traffic increase in mobile networks globally, the demand which only $4 \mathrm{G}$ technologies cannot meet. Technologies based on dynamic spectrum access (DSA), which will allow sharing and dynamic allocation of spectrum among prospective users will therefore in no measure improve spectrum utilization and efficiency. Dynamic spectrum access is identified as an important near-term opportunity for efficient spectrum usage and introduction of new wireless services (Steenkiste et al., 2009). DSA is the use of licenced spectrum opportunistically by unlicenced user when the primary user is idle or inactive and it is important in the sense that it improves the efficient utilization of radio spectrum and allows introduction of new wireless services (Steenkiste et al., 2009).

Among the DSA technologies discussed in this paper, cognitive radio seems to be the best option because of its following distinct characteristics:

i. It is capable of learning and understanding the situation regarding its environment

ii. It is able to adapt to changes in its environment

iii. From the learning, understanding and adaptation of its environment, it uses idle spectrum in a way that harmful interferences will not occur to improve spectrum efficiency.

Cognitive radios being programmable wireless devices and a disruptive technology innovation that will enable the future wireless world will soon emerge from early stage laboratory trials to become a general-purpose programmable radio that will serve as a universal platform for wireless system development, much like microprocessors have served a similar role for computation (Stenkiste, 2009). The problem of cognitive radio is the large scale deployment because of the complexity of building a network of cognitive radios (Mitran, 2008).

Cognitive radio technology is expected to improve spectrum access through: increased spectrum efficiency of licenced spectrum users; secondary markets by allowing licencees to lease their spectrum access e.g. by machine-controlled negotiation between systems; automated frequency coordination between licencees; opportunistic spectrum use by unlicenced devices while protecting incumbents from harmful interference (Kalliola, 2004).

Future work will be on how cognitive radio will be able to combine the two approaches of opportunistic usage of spectrum discussed in this paper that is, underlay and overlay, to achieve even better efficiency. It is discovered that all the spectrum sensing techniques of cognitive radios except Interference temperature model, under the classification of hierarchical access model, uses the spectrum overlay approach. In this approach, CRs referred to as secondary users (SUs) are able to opportunistically use spectrum whenever licenced users referred to as primary users (PUs) are absent. In the case when a PU signal reappears, CRs must vacate the spectrum to avoid causing interference. The disadvantage of this approach is that it does not allow SUs to coexist with the PUs. In the underlay approach used by interference temperature model, an upper interference limit is set up for a given frequency bands in a specific geographic location such that the CR users are not allowed to transmit above the set limit in order not to cause any harmful interference to the PU while using the specific band in the specific area. The disadvantage of this approach is that even when licenced users are absent, CR users cannot transmit above the set interference limit even though doing otherwise would not have caused any harmful interference.

So to further boost the efficiency of CR users, cognitive radio should optimally use the spectrum when primary users' signals are absent. That is, CRs transmitting beyond the interference limit when PU signals are absent and making sure that the transmission will not cause any harmful interference. The work will show that there will be improve spectrum efficiency and utilization under the new optimized model when compared with only either overlay or underlay.

\section{References}

[1] Ahmad Jawwad, Ben Garrison, Jim Gruen, Chris Kelly, and Hunter Pankey (2003). 4G Wireless Systems. NextGeneration Wireless Working Group.

[2] Akyildiz Ian F., Won-Yeol Lee, Vuran Mehmet C., and Mohanty Shantidev (2008). A Survey on Spectrum Management in Cognitive Radio Networks. Cognitive Radio Communications and Networks. IEEE Communications Magazine, April 2008. Pp 40 - 48.

[3] Berggren Fredrik, Olav Queseth, Jens Zander, Börje Asp, Christian Jönsson, Peter Stenumgaard, Niklas Z Kviselius, Bertil Thorngren, Urban Landmark, and Jonas Wessel (2004). Dynamic Spectrum Access. Phase 1: Scenarios and research challenges. TRITA-S3-RST-0407. ISSN 1400-9137. ISRN KTH/RST/R--04/07--SE

[4] Bliss Daniel W., Forsythe Keith W., and Chan Amanda M. (2005). MIMO Wireless Communication. Lincoln Laboratory Journal Volume 15, Number 1, 2005. pp 97-126.

[5] Cave Martin, Chris Doyle and William Webb (2007). Essentials of Modern Spectrum Management. Cambridge University Press, New York 
[6] Clancy, T.C. (2007). Achievable Capacity under the Interference Temperature Model. IEEE Communications Society subject matter experts for publication in the IEEE INFOCOM 2007 proceedings

[7] Danda, B. R. and Gongjun, Y. (2011). Spectrum Sensing Methods and Dynamic Spectrum Sharing in Cognitive Radio Networks: A Survey. International Journal of Research and Reviews in Wireless Sensor Networks. Vol. 1, No. 1.

[8] Dirk-Oliver von der Emden (2009). Efficient Use of Satellite Spectrum and Approximation of National Spectrum Fees. BR Workshop on the efficient use of the spectrum/orbit resource. Available online at http://www2.ohchr.org/english/law/resources.htm

[9] ECMA International (2005). High Rate - Ultra Wide Band (UWB): Background. Ecma/GA/2005/038.

[10] Frenzel, Louis E. (2009). Orthogonal Frequency-Division Multiplexing (OFDM): FAQ Tutorial. Retrieved from http://mobiledevdesign.com/tutorials/ofdm/

[11] Goldsmith Andrea. (2005). Wireless Communications. Cambridge University Press. Retrieved on 22-08-2011 from http://wsl.stanford.edu/ andrea/Wireless/SampleChapters.pdf

[12] Haslam, Catherine (2011). Ofcom reports $230 \%$ spectral efficiency savings with 4G. Retrieved from http://www.fiercewireless.com/europe/story/ofcom-reports-230-spectral-efficiency-savings-4g/2011-05-13

[13] Kalliola K. (2004). Spectrum Sharing and Flexible Spectrum Use. FUTURA Workshop.

[14] Lackey R.J and Upmal D.W (1995). "Speakeasy: The Military Software Radio," IEEE Communications Magazine, May 1995.

[15] Mark Norton (2007). Dynamic Spectrum Management

[16] Marshall Preston (2005). XG Communications Program Information Briefing. Presentation to the Semantic Web Applications for National Security (SWANS) Conference.

[17] Motorola (2007). Driving 4G: WiMAX \& LTE. Positioning Paper. Available on www.motorola.com

[18] Peha, Jon M. (2000). The Path Towards Efficient Coexistence in Unlicenced Spectrum. IEEE 802.16 Broadband Wireless Access Working Group. <http://ieee802.org/16>

[19] Peha, Jon M. (2008). Sharing Spectrum through Spectrum Policy Reform and Cognitive Radio. In Proceedings of the IEEE special issue on Cognitive Radio

[20] Rohde \& Schwarz, (2008). UMTS Long Term Evolution (LTE): Technology Introduction. Application Note. Retrieved online from http://www.rohde-schwarz.com

[21] Searchnetworking, (2000). Software-defined radio $\quad$ (SDR): $\quad$ Retrieved from http://searchnetworking.techtarget.com/definition/software-defined-radio

[22] Soltani Nima (2009). Comparison of Single-Carrier FDMA vs. OFDMA as 3GPP Long-Term Evolution Uplink

[23] Steenkiste Peter, Douglas Sicker, Gary Minden, Dipankar Raychaudhuri, (2009). Future Directions in Cognitive Radio Network Research. NSF Workshop Report.

[24] Taylor, S. (2003). NetTel Africa Presentation on Spectrum Management.

[25] Wang Hano, Gosan Noh, Dongkyu Kim, Sungtae Kim, and Daesik Hong (2010). Advanced Sensing Techniques of Energy Detection in Cognitive Radios. Journal of Communications and Networks, Vol.12, No.1, February 2010.

[26] Wang Li-Chun and Wang Chung-Wei (2010). Spectrum Management Techniques with QoS Provisioning in Cognitive Radio Networks. In Proceedings of IJCSIS Journal of Computer Science September 2010

[27] Williams, B.K., and Sawyer, S.C (2003). Using Information Technology: A Practical Introduction to Computers \& Communications. McGraw-Hill/Irwin, New York

[28] WiMax Forum (2006). Mobile WiMAX - Part I: A Technical Overview and Performance Evaluation.

[29] Winters, Jack H. (2003). Smart Antenna for Wireless Systems

[30] Xing Yiping, Chandramouli R., Mangold Stefan,and Sai Shankar N (2006). Dynamic Spectrum Access in Open Spectrum Wireless Networks. IEEE Journal on Selected Areas in Communications, Vol.24, No.3, March 2006. pp 626- 637.

[31] Yuguchi, K. (2008). Impact of Cognitive Radio Technology on Spectrum Management Policy. International Telecommunications Society, 17th Biennial Conference. www.canavents.com/its2008/abstracts/184.pdf

[32] Zemen T. (2008). OFDMA/SC-FDMA Basics for 3GPP LTE (E-UTRA). 